\title{
Distractor probabilities modulate flanker task performance
}

\author{
Eli Bulger ${ }^{1}$ • Barbara G. Shinn-Cunningham ${ }^{1}$ - Abigail L. Noyce ${ }^{1,2}$ \\ Accepted: 13 September 2020 / Published online: 1 November 2020 \\ (C) The Psychonomic Society, Inc. 2020
}

\begin{abstract}
Expectations about upcoming events help humans to effectively filter out potential distractors and respond more efficiently to taskrelevant inputs. While previous work has emphasized the role of expectations about task-relevant inputs, less is known about the role that expectations play in suppressing specific distractors. To address this question, we manipulated the probabilities of different flanker configurations in the Eriksen flanker task. Across four studies, we found robust evidence for sensitivity to the probability of flankers, with an approximately logarithmic relationship between the likelihood of a particular flanker configuration and the accuracy of subjects' responses. Subjects were also sensitive to length of runs of repeated targets, but minimally sensitive to length of runs of repeated flankers. Two studies used chevron stimuli, and two used letters (confirming that results generalize with greater dissimilarity between stimuli). Expanding the set of stimuli (thus reducing the dominance of any one exemplar) eliminated the effect. Our findings suggest that expectations about distractors form in response to statistical regularities at multiple timescales, and that their effects are strongest when stimuli are geometrically similar and subjects are able to respond to trials quickly. Unexpected distractors could disrupt performance, most likely via a form of attentional capture. This work demonstrates how expectations can influence attention in complex cognitive settings, and illuminates the multiple, nested factors that contribute.
\end{abstract}

Keywords Attention: Selective $\cdot$ Attention and executive control $\cdot$ Expectation

The human capacity for selective attention lets us filter relevant from irrelevant sensory inputs (James, 1890), facilitating processing of the attended stimuli (Foster, Bsales, \& Awh, 2020; Payne \& Sekuler, 2014). To effectively deploy attention, we rely on a wide range of cues, from spatial location (Johnston \& Pashler, 1990) to sensory modality (Keller, Payne, \& Sekuler, 2017; Keller \& Sekuler, 2015; Michalka, Kong, Rosen, Shinn-Cunningham, \& Somers, 2015) to specific perceptual features (Arman, Ciaramitaro, \& Boynton, 2006; Carrasco, 2011; Treue \& Martínez Trujillo, 1999). Selective attention comprises not only increased processing of task-relevant information but also increased inhibition of task-irrelevant, distracting sensory input (Neumann \& Deschepper, 1991; Tipper, 2001). This inhibition can also be established proactively, in anticipation of an event that one wishes to ignore (Aron, 2011; Braver, 2012). It is clear that expectations about distractors can improve selective attention

\section{Abigail L. Noyce}

anoyce@cmu.edu

1 Neuroscience Institute, Carnegie Mellon University, Pittsburgh, PA, USA

2 Department of Psychology, Carnegie Mellon University, Pittsburgh, PA, USA
(Noonan, Crittenden, Jensen, \& Stokes, 2018), but the contributions of different anticipatory processes that adapt to statistics across different timescales are not well understood.

Here, we modified the Eriksen flanker task (B. Eriksen \& Eriksen, 1974; C. W. Eriksen \& Eriksen, 1995) to address this question. This task is a particularly useful paradigm for understanding selective attention in the face of conflicting inputs. Subjects are instructed to report the identity of a central, target element while ignoring the presence of flanking, distractor elements. These flankers can be either congruent or incongruent with the target. Despite the subject's intention to suppress the flankers, they partially "slip through" an attentional filter (Schmidt \& Dark, 1998; Servant \& Logan, 2019), leading to longer reaction times and lower accuracy in identifying the target when the flankers and target are incongruent (Botvinick, Braver, Barch, Carter, \& Cohen, 2001; B. Eriksen \& Eriksen, 1974). This decrease in performance for incongruent relative to congruent trials is referred to as a "flanker congruency effect."

Prior work on the flanker task has demonstrated that expectations frequently affect performance. Response times decrease and accuracy increases when a trial matches preceding trials (sequential priming; Mayr, Awh, \& Laurey, 2003), and flanker congruency effects are smaller on trials following an incongruent trial or when congruent trials are rare (conflict 
adaptation; Botvinick et al., 2001; Tomat, Wendt, Luna, Michael, \& Thomas, 2020). Knowledge about forthcoming trials also reduces congruency effects (Ghinescu, Schachtman, Ramsey, Gratton, \& Fabiani, 2016; Wühr, Frings, \& Heuer, 2018) and speeds reaction times (Russeler, Kuhlicke, \& Munte, 2003). Despite the decades of literature characterizing the flanker task, no previous studies have systematically investigated how performance in the flanker task is influenced by flankers of various likelihoods. In this text, the scales of statistical regularities that potential expectations about flankers may form according to are sometimes referred to as "timescales." In our framing, separable effects of sequencing and of probability imply different timescales of the underlying cognitive or neural learning processes. Further, we typically refer to flankers as "distractors" when talking about general attentional processes.

In the classic flanker task, all configurations of target and flankers are equiprobable. While subjects may be confident that the targets and flankers are drawn from a relatively restricted set of stimuli, beyond that they can use only the spatial positions of the target and flankers to determine which elements to attend to and which to ignore. In contrast, we directly manipulated the probability of different flanker configurations to test how the predictability of distracting, nontarget stimuli influences the ability to selectively attend to a task-relevant target (Noyce \& Sekuler, 2014).

We tested two central hypotheses. First, we expected that as a flanker configuration becomes less probable, it will become harder to inhibit, leading to impaired target identification accuracy and longer response times for incongruent trials with "oddball" flankers. Second, we expected that subjects would develop expectations based on both local trial-by-trial structure, such as the number of times a given flanker configuration was repeated, as well as block-level differences in the overall probability of different flanker configurations. Both of our hypotheses were confirmed in some experimental conditions, suggesting that distractor expectations can arise via multiple cognitive mechanisms, operating on different levels of statistically driven expectation.

We conducted four experiments. In Experiment 1, we systematically varied the rarity of oddball flanker configurations to measure performance across a wide range of probabilities. In Experiment 2, we manipulated the trial sequence structure to look more systematically at interactions between local and block-level expectations. Experiment 3 was an exact replication of Experiment 2, but used letter stimuli rather than chevrons. Compared with chevrons, which map naturally to "left" and "right" button presses, use of letters both reduced similarity between the two stimulus elements and led to a more challenging and abstract stimulus-response mapping. Finally, in Experiment 4, we introduced a second set of stimuli to eliminate a potential confound between congruency and correct response. Together, the results from these experiments suggest that expectations about distractors can arise according to multiple forms of prior experience (here, we show evidence from at least two timescales), and that the degree to which they affect performance depends on how quickly and fluently subjects are able to perform the task.

\section{Experiment 1}

Experiment 1 used chevron stimuli and tested four flanker probability ratios ranging from $0.50 / 0.50$ to $0.95 / 0.05$.

\section{Methods}

All protocols of Experiments 1, 2, 3, and 4 were approved by the Institutional Review Board of Carnegie Mellon University.

\section{Subjects}

Thirty-seven members of the Carnegie Mellon University psychology subject pool (age range 18-23 years, 28 females) participated in this study, receiving partial course credit for their participation. This sample size was selected a priori to ensure we would exceed $80 \%$ power to detect medium effects (Cohen's $d=0.5$ ).

All subjects reported normal or corrected-to-normal vision and no known history of neurological disorders. Of the 37 participants recruited, one subject did not complete the session, while three additional subjects' responses did not meet our criteria for inclusion (detailed below). All analyses presented here are from the remaining 33 subjects.

\section{Stimuli and task}

The experimental paradigm consisted of a modified flanker task using chevron stimuli (B. Eriksen \& Eriksen, 1974; Noyce \& Sekuler, 2014). Figure 1 illustrates the sequence of events on each trial. A horizontal array of five chevrons was presented for $50 \mathrm{~ms}$. We refer to the center chevron as the target and the two chevrons on each side as flankers. The target chevron could be oriented to point to either the right or left. Within a trial, the four flanking chevrons all had the same orientation, which could either match the target (congruent) or be in the opposite orientation (incongruent). Subjects were instructed to respond with a key press corresponding to the orientation of the center target (left or right) after each trial presentation. Subjects were asked to respond quickly while maintaining a high degree of accuracy. Each trial was followed by a fixation cross, cueing subjects to respond. After either a response was given or 1,200 ms passed, the display went blank for a $500 \mathrm{~ms}$ intertrial interval, after which the subsequent trial began. 


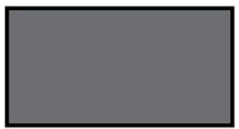

Intertrial $500 \mathrm{~ms}$

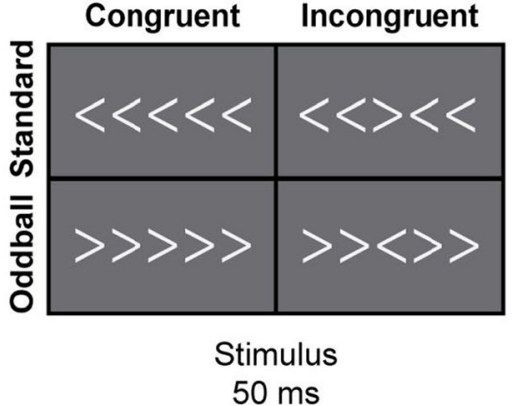

Response

$<1200 \mathrm{~ms}$
Fig. 1 On each trial, a horizontal array of five chevrons was presented for $50 \mathrm{~ms}$. Subjects were instructed to disregard the flanking chevrons and report the orientation of the central target. Although left and right target orientations were equiprobable, the orientation of the flankers was manipulated to build up different levels of predictability. In different

Stimuli were presented on a flat-panel display from a distance of approximately $60 \mathrm{~cm}$. Each chevron subtended approximately $1.5^{\circ}$ visual angle and the full array extended to an eccentricity of $4.3^{\circ}$ to the left and right of the fixation point. Chevrons were white against a gray background.

\section{Experimental design}

The goal of this study was to measure the degree to which subjects' ability to correctly report the target was affected by the probability of a given flanker configuration. For each block of trials, one flanker orientation was randomly selected (either left-pointing or rightpointing) and designated the standard orientation; the opposite orientation was designated the oddball orientation. For instance, on one block of trials, left-pointing flanker chevrons might be designated the standard and occur on $75 \%$ of trials, making right-pointing flankers, occurring on $25 \%$ of trials, the oddballs. Note that the target orientation was equiprobably left or right, regardless of flanker orientation. Similarly, congruent and incongruent trials were equiprobable. We tested four levels of probability of standard and oddball distractors: $0.50 / 0.50,0.75 / 0.25,0.90 / 0.10$, and $0.95 / 0.05$. For each, subjects completed two blocks of 320 trials, making a total of eight blocks. The order of blocks was randomized for each subject.

After each block, the experimental script provided feedback based on the accuracy of responses on those trials (after Hajcak \& Foti, 2008). If accuracy was between $75 \%$ and $90 \%$, the feedback was "You're doing great!" If accuracy was below $75 \%$, the feedback was "Try to be more accurate." If accuracy was above $90 \%$, the feedback was "Try to respond faster." Subjects were encouraged to take a brief rest break, typically 5-30 seconds, between blocks.

At the beginning of the study, subjects completed a 20-trial practice block with all stimuli equiprobable. blocks, the standard and oddball flankers could occur with respective probabilities 0.50 and $0.50,0.75$ and $0.25,0.90$ and 0.10 , or 0.95 and 0.05 . Note that either left-facing or right-facing flankers could be the standard orientation; left-facing are illustrated here

\section{Analyses}

We recorded reaction times and responses on each trial. For each condition of interest, we computed each participant's mean accuracy and median correct response time. Plots show group averages of mean accuracy and median correct response time; error bars are twice the repeated-measures standard error of the mean (Cousineau, 2005). For block-level analyses, we discarded the first 30 trials in each block to ensure that we were looking at data collected after subjects' knowledge about the probabilities had stabilized. We also discarded trials with response times faster than $200 \mathrm{~ms}$ from stimulus offset and trials that timed out (no response by $1,150 \mathrm{~ms}$ from stimulus offset). Subjects with fewer than 2,100 trials remaining after this step were excluded from all analyses as described above. Our final data set comprised 33 subjects who gave valid responses on more than 2,100 trials or approximately $90 \%$ of trials (mean $=2,236$; range: $2,107-2,290$ trials). The percentage of excluded trials based on trial conditions was standard congruent, $4.59 \%$; oddball-congruent, $4.76 \%$; standard-incongruent, 4.38\%; oddball-incongruent, $4.30 \%$.

We performed post hoc analyses of serial order effects within sequences of trials by considering the first trial after a run of either repeated target orientations or repeated flanker orientations.

\section{Results}

We manipulated the probability of flanker configurations in the Eriksen flanker task. Figure 2 summarizes response accuracy and median correct response time. Proportion correct showed a classic flanker congruency effect for both the standard and oddball flanker orientations, with values higher on congruent trials than on incongruent trials. Overall, accuracy was highest on standard-congruent trials $(0.931, S D=0.044)$ and on oddball-congruent trials $(0.931, S D=0.064)$, next highest on standard-incongruent trials $(0.852, S D=0.059)$, and lowest on oddball-incongruent trials $(0.722, S D=$ 
a

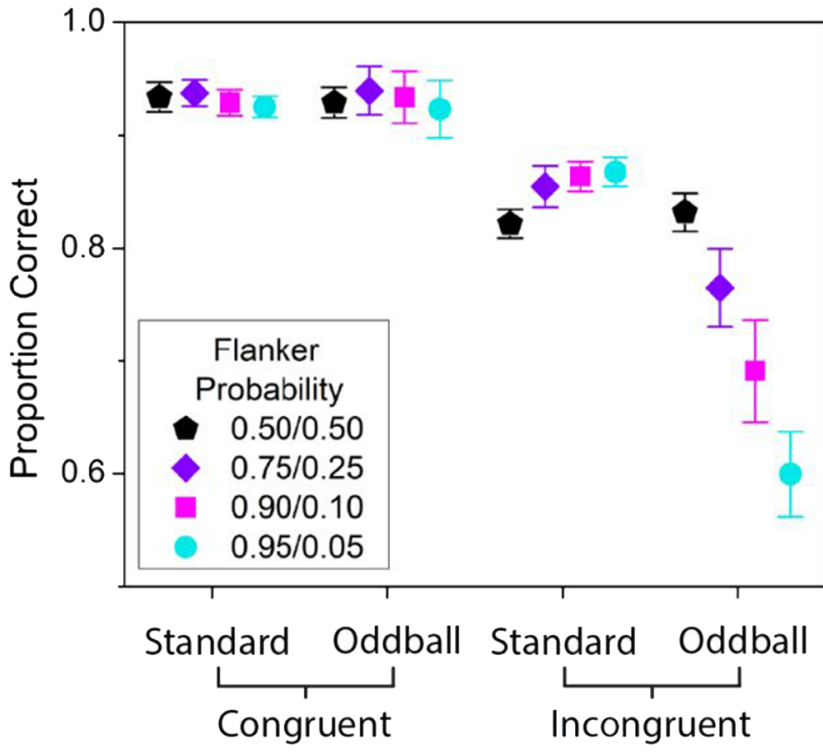

Fig. 2 Accuracy and response time for all conditions. a Proportion correct for each trial type (standard-congruent, standard-incongruent, oddball-congruent, oddball-incongruent) by flanker probability level $(0.50 / 0.50,0.75 / 0.25,0.90 / 0.10,0.95 / 0.05)$. b Median response time for

0.142). For oddball trials, the congruency effect increased as oddballs become rarer, driven mostly by accuracy on the incongruent trials becoming worse as their incidence decreased. A small change in the other direction occurred for standard flankers, with the congruency effect decreasing slightly as oddballs became rarer.

Response time showed a corresponding pattern, with subjects responding more quickly on congruent than on incongruent trials. Overall, responses were fastest on standardcongruent trials $(338 \mathrm{~ms}, S D=41 \mathrm{~ms})$ and oddballcongruent trials ( $339 \mathrm{~ms}, S D=44 \mathrm{~ms})$, next fastest on standard-incongruent trials ( $374 \mathrm{~ms}, S D=50 \mathrm{~ms})$, and slowest on oddball-incongruent trials ( $408 \mathrm{~ms}, S D=69 \mathrm{~ms}$ ).

For each trial type (standard congruent, standard-incongruent, oddball-congruent, and oddball-incongruent), two repeated-measures analyses of variance (ANOVAs) tested whether flanker probability affected accuracy or response time. For standard-congruent and oddballcongruent trials, there was no significant effect of flanker probability on either accuracy, standard-congruent, $F(3,96),=1.157, \mathrm{p}=.3304$; oddball-congruent, $F(3$, $96)=.549, p=.6501$, or response time, standard-congruent, $F(3,96)=0.552, p=.6477$; oddball-congruent, $F(3,96)=.162, p=.9218$. For standard-incongruent trials, there was an effect of flanker probability on accuracy, $F(3,96)=6.759, p=.0004$, but not on response time, $F(3,96)=1.576, p=.2003$, while on oddball-incongruent trials there was a effect of flanker probability on both accuracy, $F(3,96)=33.643, p<$ .0001 , and response time, $F(3,96)=2.847, p=.0416$. b

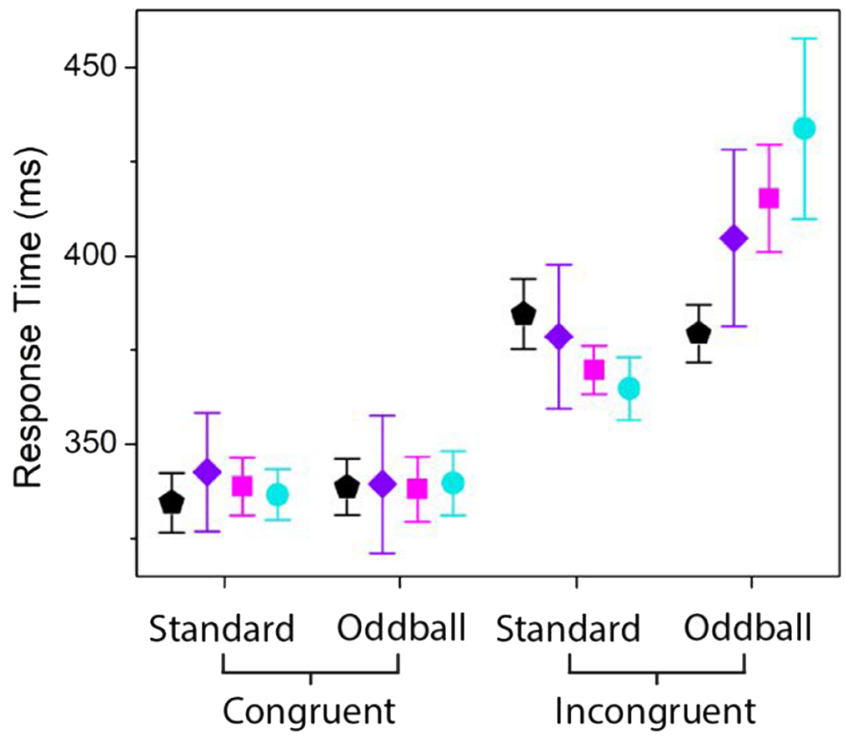

correct responses of each trial type by flanker probability level. Error bars are twice the repeated-measures standard error of the mean (Cousineau, 2005). Data points are displaced along the $x$-axis to aid visualization

Post hoc $t$ tests confirmed that performance on oddballincongruent trials was significantly different at each level of flanker probability. Subjects were slower and less accurate for incongruent trials with flanker probability 0.25 than on those with flanker probability 0.50 , accuracy: paired, $t(32)=3.722$, $p=.0008$; response time: paired $t(32)=24.452, p<.0001$, slower and less accurate on trials with flanker probability of 0.10 than on those with flanker probability 0.25 , accuracy: paired $t(32)=3.182, p=.0032$; median correct response time: paired $t(32)=15.626, p<.0001$, and slower and less accurate on trials with flanker probability 0.05 than on those with 0.10 , accuracy: paired $t(32)=2.924, p=.0063$; median correct response time: paired $t(32)=2.924, p<.0001$.

Figure 3a replots accuracy on incongruent trials for all flanker probabilities (both standard and oddball). Proportion correct logarithmically increased with flanker probability $\left[\mathrm{pCorrect}=.876+.069 \times \ln (\mathrm{pFlanker}-.031)\right.$, Adj. $R^{2}=$ .504]. Response time somewhat decreased with flanker probability [RT $=424.24-66.74 \times$ pFlanker, Adj. $\left.R^{2}=.114\right]$. Response time was slightly better fitted by a multiparameter exponential model, but both fits are weak enough that we do not make a claim about the shape of the relationship. Figure $3 \mathrm{~b}$ shows accuracy on incongruent trials by decile of response time (only points with response times under $600 \mathrm{~ms}$ are shown) and flanker probability. For all flanker probabilities, accuracy is lowest at fast response times and increases as response time increases. This effect interacts with the probability of the flanker configuration: As oddball flankers become rarer, subjects' responses to them become both slower and less accurate. 
a

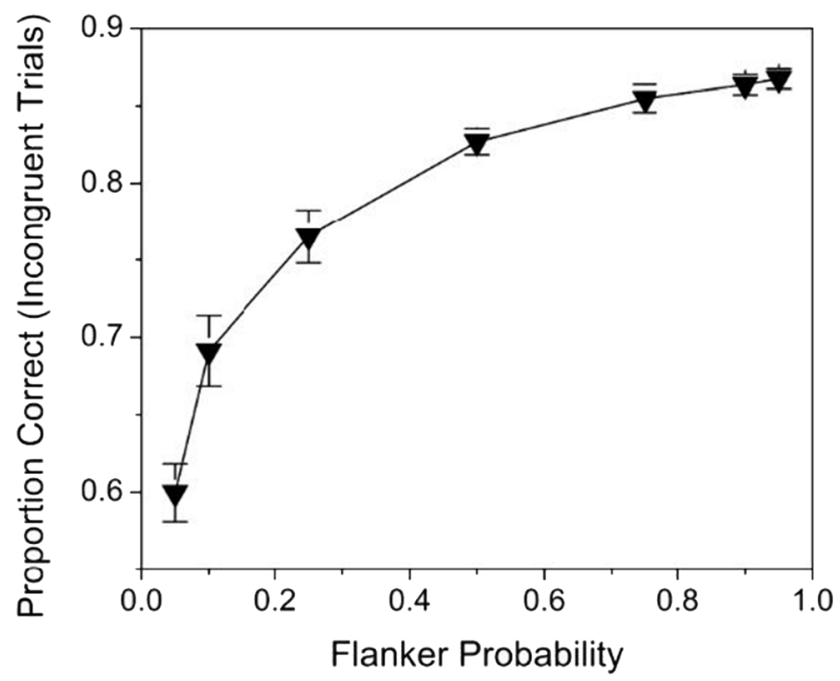

b

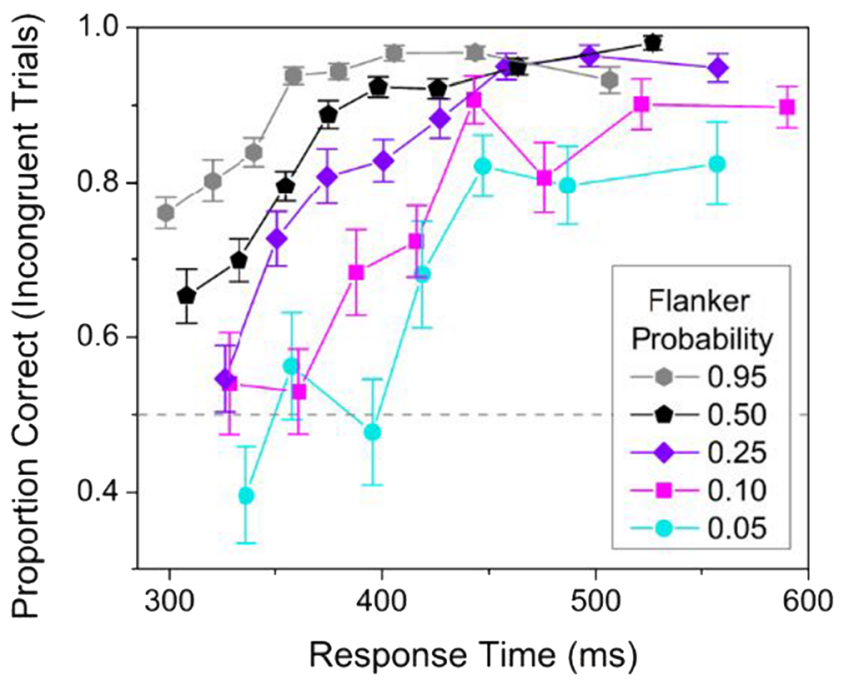

Fig. 3 a Accuracy on incongruent trials (replotted from Fig. 2). There is a strong logarithmic-scale relationship between flanker probability and proportion correct response. b Incongruent trials binned by response time. Proportion correct was calculated for each response time decile of each condition $(0.75$ and 0.90 flanker probability data followed similar trends and are omitted here for visualization, as are points with response time greater than $600 \mathrm{~ms}$ ). The dotted gray line marks chance accuracy. Error bars are twice the repeated-measures standard error of the mean. Data points are not displaced along the $x$-axes of either plot

To test the effect of local trial-by-trial structure, we looked at accuracy on trials immediately following an extended run of trials in which the target orientation or flanker orientation was repeated (see Fig. 4). To improve signal-to-noise ratios when visualizing sequences of repeated flanker orientations, we binned together sequences of progressively longer lengths $(2,3-7,8-15,16-31)$. Bins that did not include at least one trial from at least 30 subjects were discarded at this step.

Runs of repeated targets led to decreased accuracy on the subsequent trial; however, there was no consistent effect of flanker probability (see Fig. 4a). Runs of trials with the same a

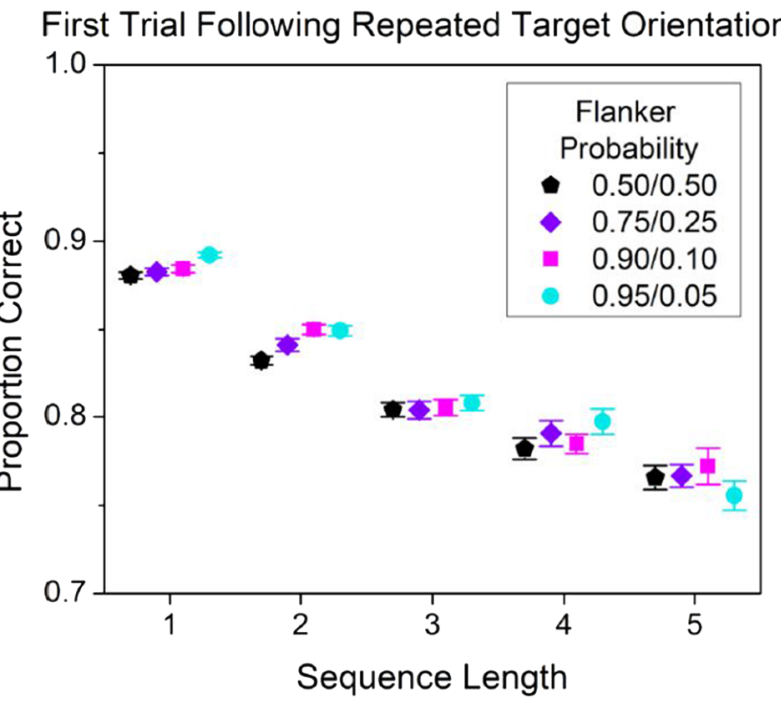

b

First Trial Following Repeated Flanker Orientations

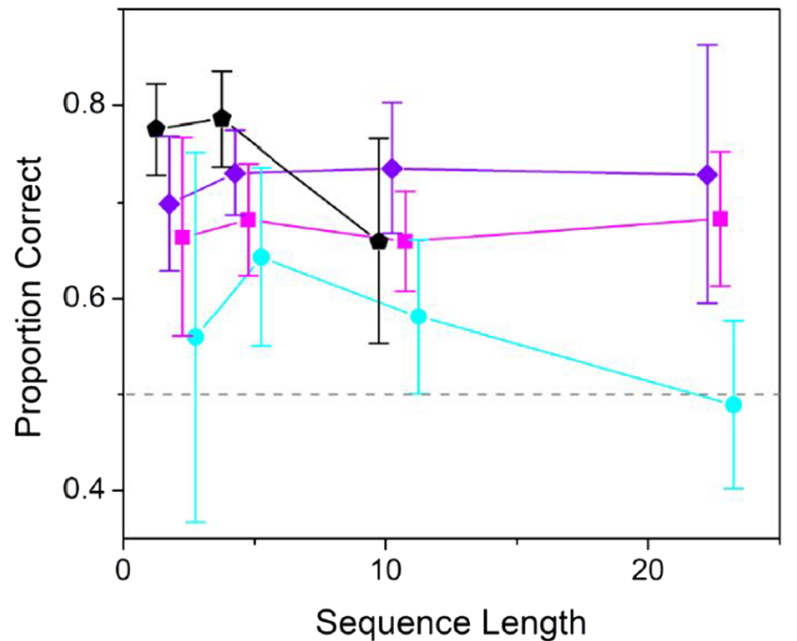

Fig. 4 Effects of local-trial-by-trial structure. a Accuracy on the first trial following a sequence of repeated target orientations. Data points are displaced along the $x$-axis to aid visualization. b Accuracy on the first oddball-incongruent trial following a sequence of repeated flanker orientations. Sequence lengths are grouped together to improve estimation accuracy; longer length sequences are less likely, so are therefore averaged over a greater set of sequence lengths (lengths 2, 3-7, 8-15, 16-31). The dotted gray line marks chance accuracy. Error bars are twice the repeated-measures standard error of the mean

flanker orientation, on the other hand, seemed to remain stable while the effect of block-level flanker probability was pronounced. Accuracy was lowest when oddballs were least likely (probability 0.05 ) and highest when oddballs were most likely (probability 0.50 ), even for identical preceding sequence lengths (i.e., identical local trial-by-trial structure).

We fit mixed-effects logistic models with subjects as a random intercept to test effects of sequence length and probability level, and found that probability level was the only significant predictor (corrected $p<.0001$, Holm-Bonferroni 
corrected for six comparisons). Although sequence length significantly predicted proportion correct when probability level was excluded from the model (corrected $p<.0001$ ), models including probability level were not improved by adding either sequence length or the probability by sequence length interaction.

Similar analyses of response times did not find any significant effects. We also investigated a number of other potential order effects, including over the course of blocks, within sequences of repeated flankers or targets, and across the entire experimental session, but found no consistent effects and thus do not report them here.

\section{Discussion}

We modified the Eriksen flanker task to investigate the effect of probable and improbable visual distractors on selective attention. Our results display the typical flanker congruency effect, where subjects respond faster and more accurately on congruent trials than on incongruent trials (Botvinick et al., 2001; B. Eriksen \& Eriksen, 1974). We manipulated the likelihood of flanker configuration within experimental blocks (left-pointing versus right-pointing) to test whether expectations about distracting stimuli influenced performance. When confronted with incongruent trials with a rare flanker configuration, participants showed a clear pattern of flanker probability modulating performance (see Fig. 2). Responses on incongruent trials were slowest and least accurate when oddball flankers were most unexpected (5\% of trials) and improved approximately logarithmically as flankers became more predictable (see Fig. 3a). Subjects were both slower and less accurate on incongruent trials with less-probable distractors (see Fig. 3b). Finally, our analysis of sequential effects after runs of successive target or flanker identities showed that performance is shaped by both block-level statistical regularities and local repetition sequences (see Fig. 4).

Participants appear to be able to use their experience in the task to develop attentional templates that potentially let them more effectively inhibit expected distractors, facilitating correct responses on standard-incongruent trials as opposed to oddball-incongruent trials. This process could explain these results, and it is often referred to as expectation-mediated distractor inhibition (Noonan et al., 2018; van Moorselaar, 2020). Most previous work on expectation-mediated distractor inhibition used paradigms that explicitly cued subjects to expect a distractor, or to expect a particular feature such as location or color (Awh, Matsukura, \& Serences, 2003; Chao, 2011; Ruff \& Driver, 2006). Probabilistic expectations about the location of distractors or distractor-target configurations can also facilitate visual search (Goschy, Bakos, Müller, \& Zehetleitner, 2014; Leber, Gwinn, Hong, \& O'Toole, 2016; Tseng, Tzengô, \& Hungô, 2011; Wang \& Theeuwes, 2018). Interestingly, although we previously found that flanker probability modulated performance in congruent trials in addition to incongruent trials (Noyce \& Sekuler, 2014), here, we see little evidence of flanker probability modulating congruent trials. This may be because our subjects were already at ceiling, or because our experimental blocks were slightly shorter than in our previous study.

To shed light on how local trial-by-trial structure and block-level probabilities each affected the results, we analyzed performance after runs of repeated target or flanker identities (see Fig. 4). This analysis suggested that performance is shaped by both block-level statistical regularities and local trial-by-trial structure. Short runs of repeated targets led to decreased accuracy on the first subsequent trial (consistent with Ferdinand, Mecklinger, \& Kray, 2008; van Moorselaar \& Slagter, 2019). Runs of repeated flankers, on the other hand, showed a strong effect of flanker probability, with lower accuracy for improbable than probable flanker orientations even when local trial structure was identical.

The post hoc nature of this analysis meant that runs of trials - especially long runs - did not occur frequently or consistently. In our subsequent experiments, we manipulated the trial structure to ensure that a sufficient number of long runs occurred in all conditions for all subjects. Experiment 2 reprises the chevron stimuli used in Experiment 1. Experiment 3 replicates the design and structure of Experiment 2, but uses letter stimuli rather than chevrons.

\section{Experiments 2 and 3}

Experiment 2 used chevron stimuli and tested flanker probabilities of $0.50 / 0.50$ and $0.90 / 0.10$. The blocks of trials were constructed to include long runs of repeated flankers. Experiment 3 was identical to Experiment 2, except that the stimuli were letters.

\section{Methods}

\section{Subjects}

Subjects were recruited from Prolific, an online participant recruitment service (http://prolific.co). For Experiment 2, 50 subjects were recruited (ages 18-23 years, 12 females). Two subjects' responses did not meet our criteria for inclusion (see Analyses). All analyses presented for Experiment 2 are from the remaining 48 subjects.

For Experiment 3, 50 subjects were recruited online from the Prolific participant pool (ages 18-23, 19 females). Four subjects' responses did not meet our criteria for inclusion. All analyses presented for Experiment 3 are from the remaining 46 subjects. For both experiments, all subjects reported normal or corrected-to-normal vision. 
Sample sizes were selected a priori to ensure we would exceed $80 \%$ power to detect medium effects (Cohen's $d=$ $0.5)$, even with potentially high rates of unusable data.

\section{Stimuli and task}

All experiment presentation and data collection occurred online using Gorilla (Anwyl-Irvine, 2020; http://gorilla. sc). Subjects reported using a desktop or laptop computer and web browser to complete the task. Figure 1a illustrates the sequence of events on a single trial.

Experiment 2 The task and stimuli were very similar to those of Experiment 1. Black chevrons were displayed on a white background for $50 \mathrm{~ms}$, and subjects were instructed to report the orientation of the target chevron (see Fig. 1b). Subjects had 1,950 ms from stimulus offset in which to make their response, followed by a 700-ms intertrial interval.

Experiment 3 All experimental timing was identical to Experiment 2. The target and flankers were letters (displayed in black on a white background), with the same structure of one target and four identical flankers. As the letters were " $\mathrm{C}$ " and "H," the four stimulus configurations were $\mathrm{HHHHH}$, $\mathrm{CCCCC}, \mathrm{HHCHH}$, and CCHCC. Subjects were instructed to press a left key when the target was a " $\mathrm{C}$ " and a right key when the target was an " $\mathrm{H}$ ". Figure 5 shows the trial structure and stimuli used in Experiments 2 and 3.

\section{Experimental design}

The experimental structure was the same for both Experiments 2 and 3. As in Experiment 1, we varied the probability of different flanker configurations by balancing across subjects the set that was standard and the set that was oddball. We tested two levels of probability of standard and oddball distractors: $0.50 / 0.50$ and $0.90 / 0.10$.

Subjects first completed at least 40 practice trials in which all stimuli were equiprobable. They then completed five experimental blocks of $0.50 / 0.50$ flanker probability, followed by five experimental blocks of 0.90/0.10 flanker probability (with the same stimulus designated as the standard in each). Each block contained 100 trials. Subjects were encouraged to take short breaks in between blocks. The flanker configuration selected as standard was balanced across subjects. Left-response and right-response target stimuli were equiprobable. Blocks of trials were pregenerated (using Python scripts) to control the number and length of standard trial sequences preceding oddball-incongruent trials. In each block, at least one instance each of five different standard trial sequences (lengths: 2, 4, 7, 11, and 16) preceded an oddball-incongruent trial. Pregenerated blocks of trials were randomized across subjects.

\section{Analyses}

We recorded reaction times and responses on each trial. Measures are computed as in Experiment 1. We discarded a

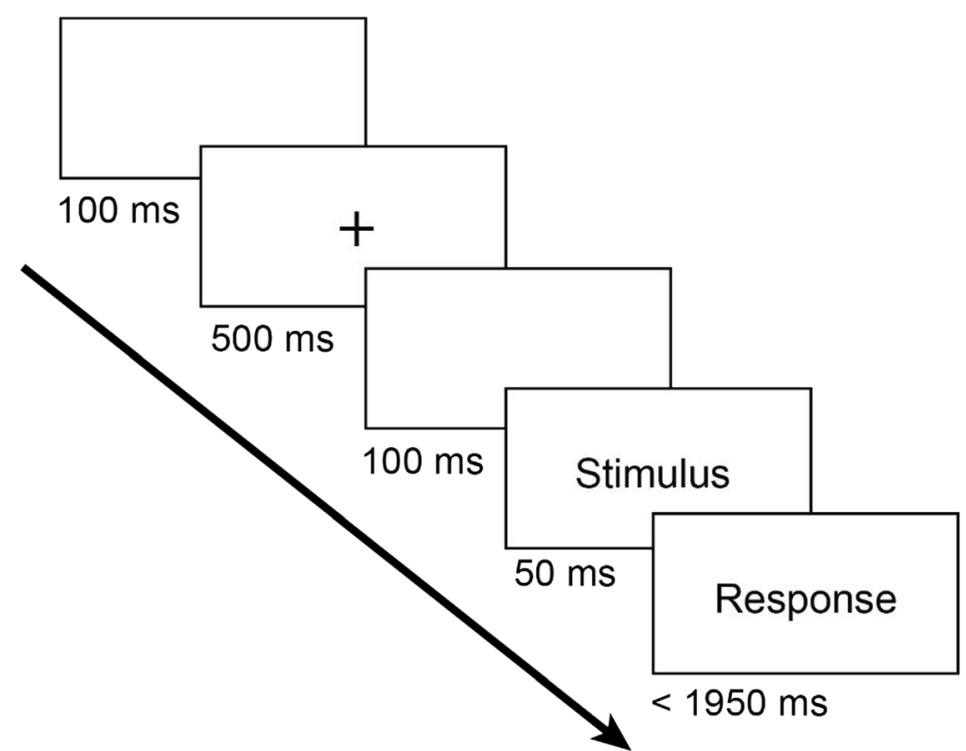

b

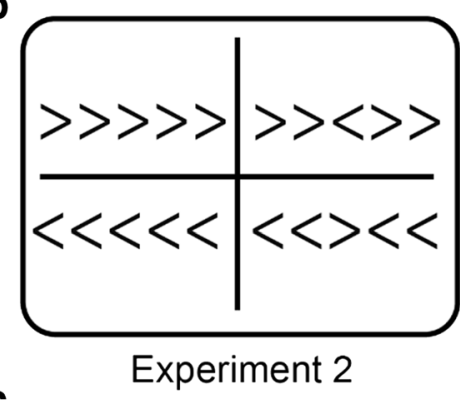

c

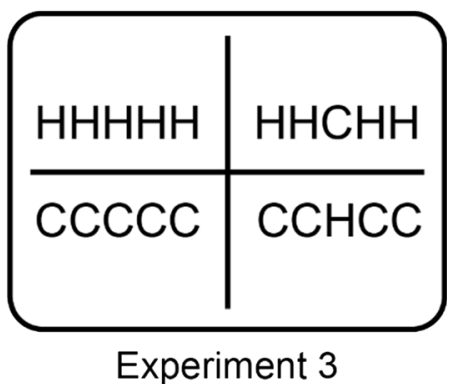

Fig. 5 Trial structure and stimuli for Experiments 2 and 3. a On each trial, a 700-ms intertrial interval was followed by a 50-ms flankers display and up to 1,950 ms for subjects to make a response. b Chevron stimuli used in Experiment 2. c Letter stimuli used in Experiment 3 
trials with response times below $200 \mathrm{~ms}$ from stimulus offset and trials that timed out (no response by $1,950 \mathrm{~ms}$ from stimulus offset). Subjects with fewer than $85 \%$ of trials remaining after this step were excluded from all analyses.

For Experiment 2, our final data set comprised 48 subjects who gave valid responses on more than 850 of 1,000 trials (mean = 983 trials, range: 899-1,000). The percentage of excluded trials based on trial conditions was standard-congruent, $1.98 \%$; oddball-congruent, 2.09\%; standard-incongruent, 1.84\%; oddball-incongruent, $2.14 \%$. For Experiment 3, our final data set comprised 46 subjects who gave valid responses on more than 850 of 1,000 trials (mean $=981$ trials, range: $888-1,000)$. The percentage of excluded trials based on trial conditions was standard-congruent, $1.58 \%$; oddball-congruent, $1.97 \%$; standard-incongruent, $2.32 \%$; oddball-incongruent, $1.73 \%$.

a

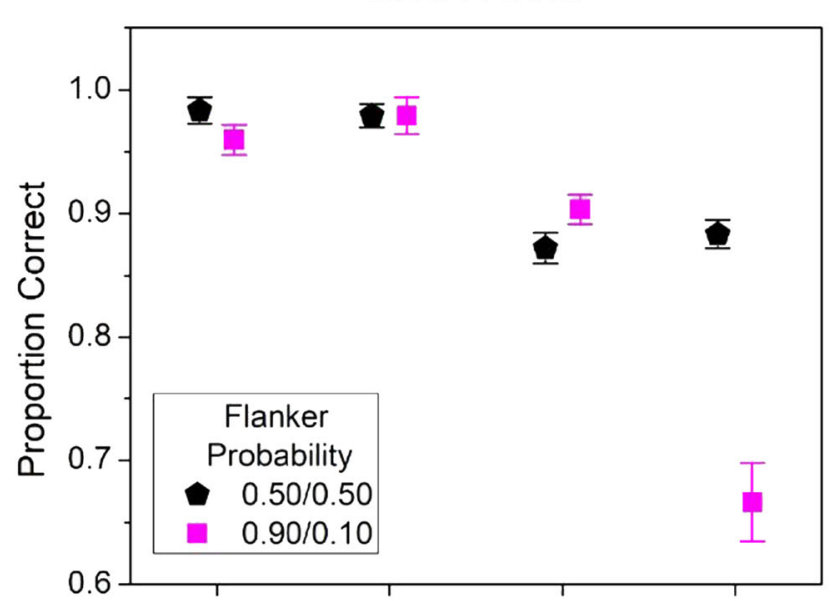

C

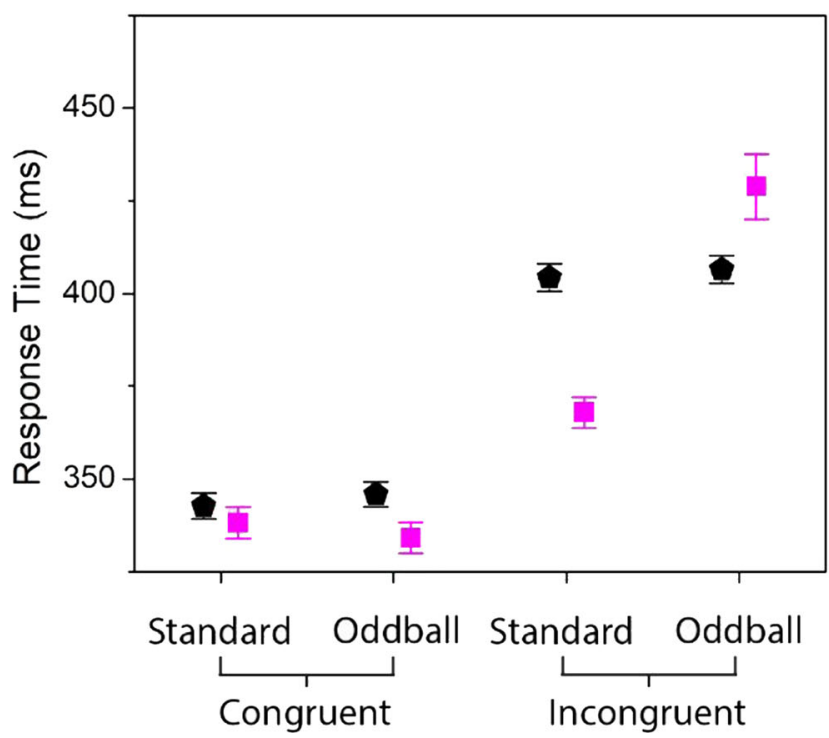

Fig. 6 Accuracy and correct response time for all conditions, by flanker probability level. a Experiment 2 (chevrons) proportion correct for each trial type. b Experiment 3 (letters) proportion correct for each trial type. c

\section{Results}

We modified Experiment 1 by explicitly generating runs of standard trials preceding oddball-incongruent trials. Our goals were to collect more reliable data about sequence lengths and block-level probability, and to assess whether these results were dependent on the details of the stimuli used. Figure 6 summarizes response accuracy and median correct response time for Experiments 2 and 3.

Experiment 2 In both Experiment 2 and Experiment 3, we observed a flanker congruency effect, which was largest for oddball trials in the $0.90 / 0.10$ condition. Accuracy was highest on oddball-congruent trials $(0.979, S D=0.018 \mathrm{~ms})$ and on standard-congruent trials $(0.971, S D=0.016)$, next

b

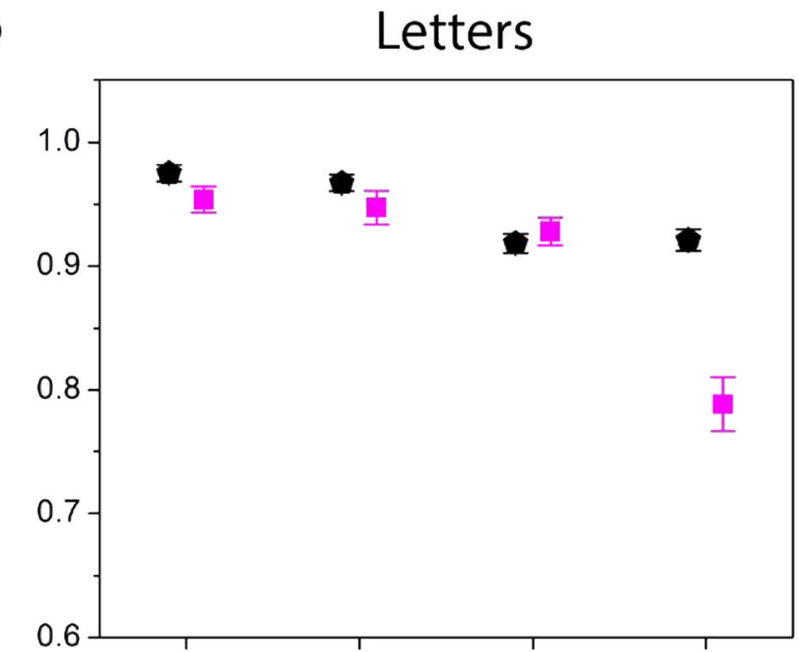

d

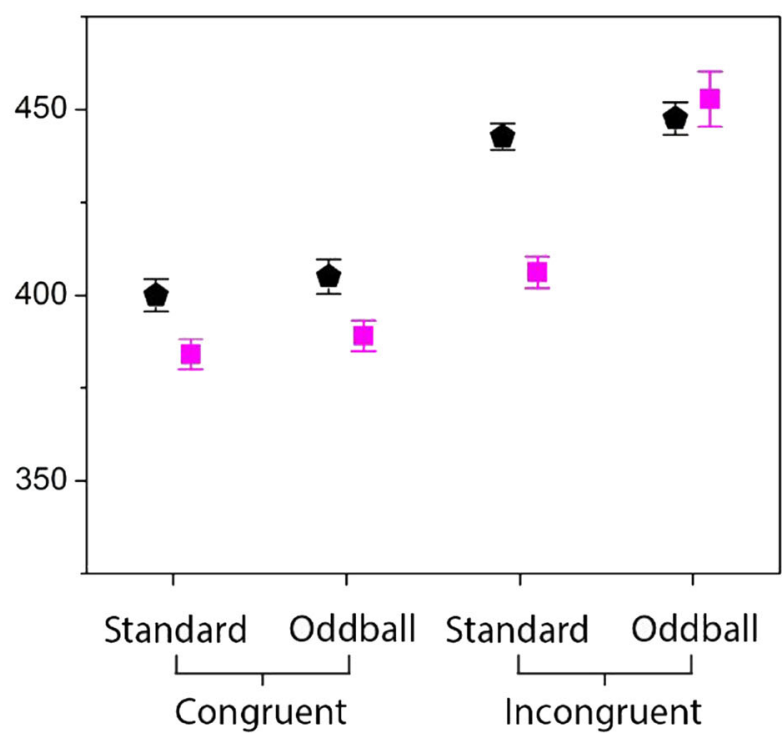

Experiment 2 (chevrons) correct response time for each trial type. d Experiment 3 (letters) correct response time. Error bars are twice the repeated-measures standard error of the mean 
highest on standard-incongruent trials $(0.888, S D=0.051)$, and lowest on oddball-incongruent trials $(0.775, S D=$ 0.084). Similarly, responses were fastest on oddballcongruent trials $(340 \mathrm{~ms}, S D=20 \mathrm{~ms})$ and standardcongruent trials $(340 \mathrm{~ms}, S D=21 \mathrm{~ms})$, next fastest on standard-incongruent trials ( $386 \mathrm{~ms}, S D=24 \mathrm{~ms}$ ), and slowest on oddball-incongruent trials ( $418 \mathrm{~ms}, S D=31 \mathrm{~ms}$ ).

Performance on $0.50 / 0.50$ incongruent trials was significantly different from performance on $0.90 / 0.10$ incongruent trials. Subjects were significantly slower and less accurate on oddballincongruent trials (flanker probability 0.10 ) than on incongruent trials with flanker probability 0.50 , accuracy: paired $t(47)=$ 8.323, $p<.0001$; response time: paired $t(47)=2.024, \mathrm{p}=$ .0488 . Subjects were faster and more accurate on standardincongruent trials (flanker probability 0.10 ) than on incongruent trials with flanker probability 0.50 , accuracy: paired $t(47)=$ 2.279, $p=.0273$; response time: paired $t(47)=4.069, p=.0002$.

Experiment 3 Again, accuracy was highest on standardcongruent trials $(0.964, S D=0.021)$ and on oddball-congruent trials $(0.957, S D=0.033 \mathrm{~ms})$, next highest on standardincongruent trials $(0.923, S D=0.036)$, and lowest on oddballincongruent trials $(0.855, S D=0.064)$. Subjects responded more quickly on congruent than on incongruent trials. Overall, responses were fastest on standard-congruent trials $(392 \mathrm{~ms}, S D$ $=39 \mathrm{~ms})$ and oddball-congruent trials $(397 \mathrm{~ms}, S D=41 \mathrm{~ms})$, next fastest on standard-incongruent trials ( $424 \mathrm{~ms}, S D=41 \mathrm{~ms}$ ), and slowest on oddball-incongruent trials ( $450 \mathrm{~ms}, S D=45 \mathrm{~ms}$ ).

Accuracy on $0.50 / 0.50$ incongruent trials was significantly different from accuracy on $0.90 / 0.10$ incongruent trials. Subjects were significantly less accurate (but not significantly slower) on oddball-incongruent trials (flanker probability

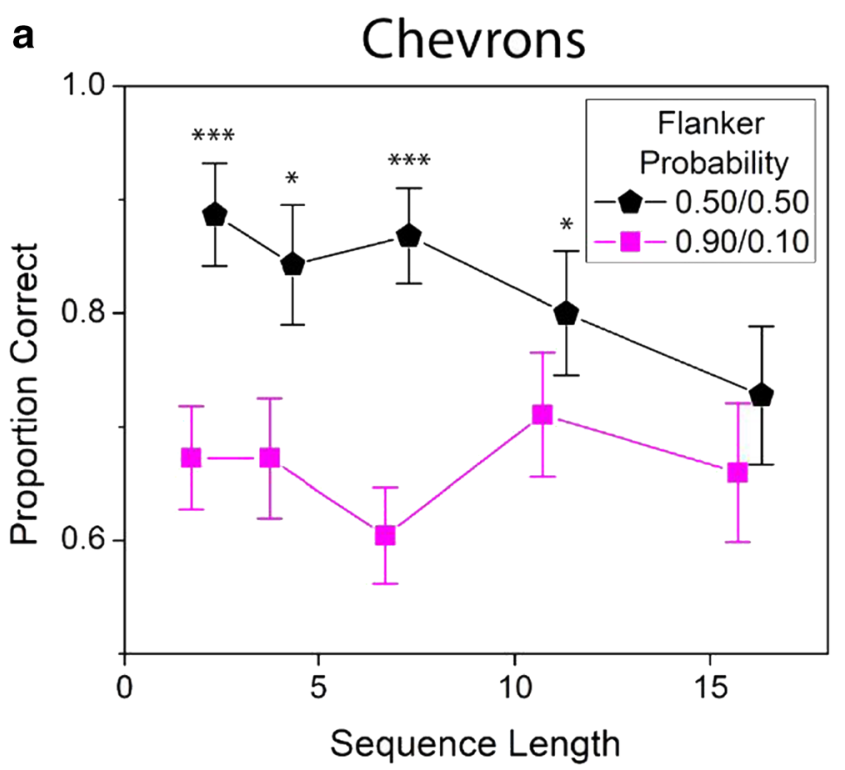

Fig. 7 Accuracy on oddball-incongruent trials following runs of standard trials. a Data from Experiment 2 (chevron stimuli). b Data from Experiment 3 (letter stimuli). Error bars are twice the repeated-measures
$0.10)$ than on trials with flanker probability 0.50 , accuracy: paired $t(45)=7.043, p<.0001$; response time: paired $t(45)$ $=0.371, p=.7121$. Subjects were faster but not significantly more accurate on standard-incongruent trials with flanker probability 0.90 than on those with flanker probability 0.50 , accuracy: paired $t(45)=0.879, p=.3839$; response time: paired $t(45)=2.948, p=.0051$.

In general, response times in Experiment 3 (with letter stimuli) are 25-50-ms greater than those with in Experiment 2 (chevron stimuli). In both experiments, response times on the $0.90 / 0.10$ trials is approximately $5-20$-ms faster than on the $0.50 / 0.50$ trials (most evident in congruent trials); this is likely due to order effects across the experiment.

Runs of standard trials In both Experiments 2 and 3, runs of standard trials followed by an oddball-incongruent trial were explicitly generated. Figure 7 summarizes the relationship between standard trial sequence length and block-level flanker probability using stimuli from Experiment 2 and Experiment 3. In both experiments, subjects respond correctly to the oddball-incongruent trial more frequently in blocks with $0.50 / 0.50$ flanker probabilities than in blocks with $0.90 / 0.10$ flanker probabilities, although the effect is larger in Experiment 2 (chevrons). At long runs (16 preceding trials), the proportion correct in $0.50 / 0.50$ blocks decreases to a rate similar to that of $0.90 / 0.10$ blocks.

We fit mixed-effects logistic models with subjects as a random intercept to test for effects of sequence length and probability level. For Experiment 2 (chevrons), sequence length, flanker probability, and the interaction between them, all contributed significantly to accuracy (corrected $p<.05$ at each model-fitting step; Holm-Bonferroni corrected for six

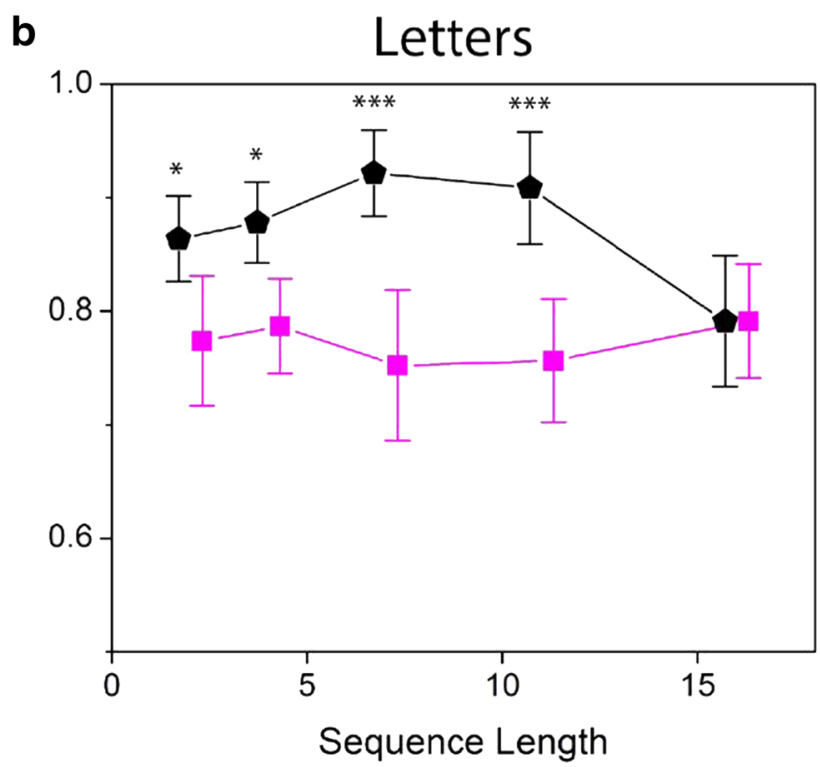

standard error of the mean. Significant differences between $0.50 / 0.50$ and $0.90 / 0.10$ probability levels are denoted with $* p<.05 ; * * * p<.001$ 
comparisons). For Experiment 3 (letters), only flanker probability contributed significantly (corrected $p<.0001$ ).

\section{Discussion}

In Experiments 2 and 3, we generated sequences of trials that included long runs of standard flanker orientations followed by an oddball-incongruent trial. This allowed us to more fairly estimate the effects of a sequence of preceding trials. In Experiment 2, which used chevron stimuli, both sequence length and oddball probability significantly influenced accuracy. In Experiment 3, which used letter stimuli, only oddball probability contributed significantly. In both experiments, we again observed a flanker congruency effect that was modulated by flanker probability.

Because Experiments 2 and 3 had identical structure and differed only in their stimuli, we were able to directly assess effects of chevrons versus letters. Two potential differences between the stimuli could contribute to our results. Left-facing and right-facing chevrons are more geometrically similar to one another than are the letters "C" and " $\mathrm{H}$," and thus may be more difficult to attentionally segregate in a crowded display. On the other hand, chevrons are naturally directional, and map intuitively to the left and right key presses; remembering that "C" and " $\mathrm{H}$ " map to left and right key presses, respectively, requires a more abstract mapping from symbol to response and is thus more demanding.

We observed that response times in Experiment 3 were generally higher than those in Experiment 2 (see Fig. 6c-d), although in the critical $0.90 / 0.10$ oddball-incongruent condition, there was no significant difference between the two experiments. Accuracy in most conditions was similar between the two experiments, except for the $0.90 / 0.10$ oddball-incongruent condition, which had significantly lower accuracy on Experiment 2 than on Experiment 3 (see Fig 6a-b). These results suggest that in Experiment 3, subjects took more time to map target letters to key-press responses, and that their performance was less disrupted by unexpected configurations. This could be due to either the pattern of slower, more effortful and less automatic responses (which may disrupt expectation formation) or to the geometric dissimilarity between " $\mathrm{C}$ " and " $\mathrm{H}$ " (which may reduce the degree to which oddball flankers are able to interfere with target selection, due to better perceptual segregation between target and flankers). One interesting question for future investigations will be further separating these aspects.

The principal motivation for Experiments 2 and 3 was to look more closely at the effects of local-scale serial order and block-level flanker probability to understand the dynamics of performance modulation by distractor probability. Our results are consistent with those of Experiment 1: The experimental structure over multiple scales can affect performance. For both chevron and letter stimuli, we observed that accuracy on oddball-incongruent trials was greater in $0.90 / 0.10$ flanker probability blocks than in $0.50 / 0.50$ blocks, confirming an effect of block-level statistical structure. Sequence length significantly contributed to lower accuracy for chevron stimuli, but not for letter stimuli, suggesting that subjects are sensitive to local trial-by-trial structure but only under some conditions. The degree of automaticity of subjects' responses may be a key difference between Experiments 2 and 3 (Cohen, ServanSchreiber, \& Mcclelland, 1992).

Finally, we note that at the longest sequence length tested, 16 preceding trials, accuracy in $0.50 / 0.50$ blocks decreased to approximately match that in $0.90 / 0.10$ blocks. These data suggest that even when local trial-by-trial structure is not contributing measurably to short-term expectations, these longer sequences may start to activate a form of learning or expectation closely related to that for block-level statistics.

One potential confound in these first three experiments is that congruency and target identity covary. A $0.90 / 0.10$ block of trials will have 45 standard-congruent trials and only 5 oddballcongruent trials. A subject using a strategy of reporting congruency rather than target identity would thus be able to perform quite well. We designed Experiment 4 to assess whether our results would replicate in the presence of a more complex stimulus set that controlled for this potential confound.

\section{Experiment 4}

Experiment 4 expanded the stimulus set to include eight rather than four stimulus configurations. This allowed us to control the relationship between trial congruency and correct response which was confounded in other experiments.

\section{Methods}

Experiment 4 used methods identical to those of Experiments 2 and 3, except as noted below.

\section{Subjects}

Fifty-four subjects were recruited from Prolific (ages 18-23 years, 18 females). All subjects reported normal or correctedto-normal vision. Six subjects' responses did not meet our criteria for inclusion; all analyses presented here are from the remaining 48 subjects.

\section{Stimuli and task}

The events and timing within each trial was identical to that of Experiments 2 and 3. The stimuli were made up of the letters "C," "H," "S," and "K." Subjects were instructed to make a left key press when the target was "C" or "S" and a right key press when the target was "H" or "K." Each target was presented with four flanking letters, producing either congruent (CCCCC, 
a

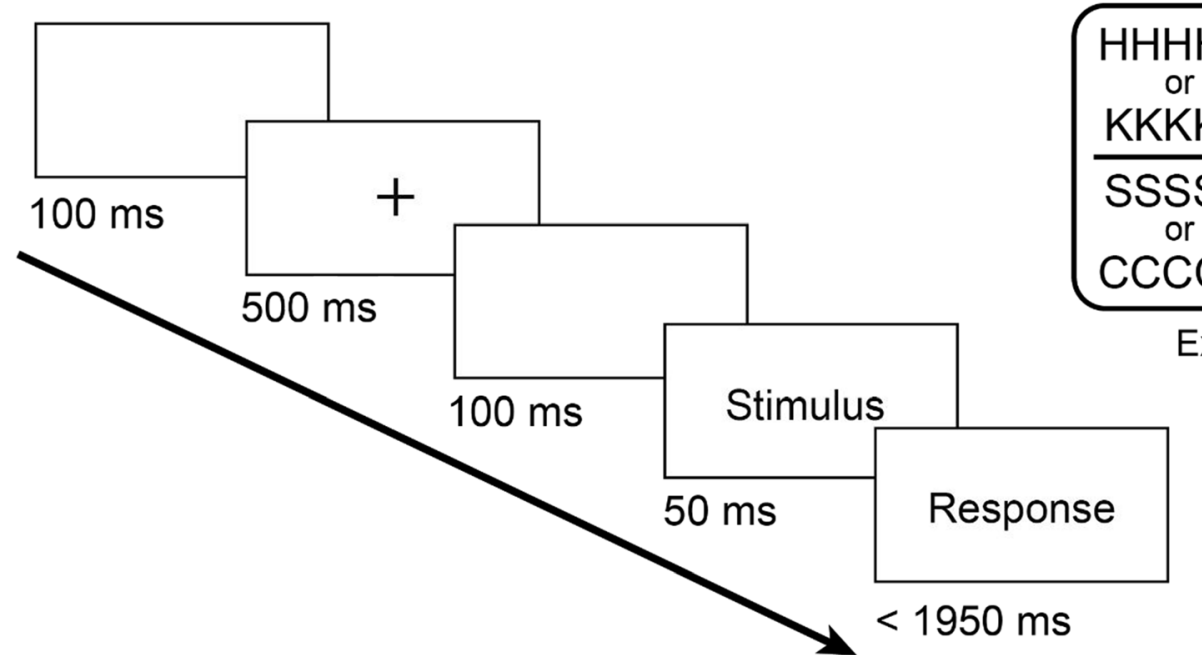

b
Fig. 8 Trial structure and stimuli where (a) shows the flow of screens presented during each trial and (b) shows the stimuli used. The upper and lower quadrants of (b) show stimuli with flankers corresponding to respective "right" and "left" directions, and the left and right quadrants respectively show congruent and incongruent stimuli. Left and right target orientations were equiprobable. Stimuli on either the top or bottom of each quadrant were equiprobably standard or oddball, to balance target identity with congruency. For example, if $\mathrm{HHHHH}$, $\mathrm{HHCHH}$, SSSSS, and SSKSS were standard configurations, then oddball configurations would be KKKKK, KKSKK, CCCCC, and CCHCC. Standard and oddball flankers occurred with respective probabilities 0.50 and 0.50 , or 0.90 and 0.10
HHHHH, SSSSS, KKKKK) or response-incongruent (HHCHH, CCHCC, KKSKK, SSKSS) stimuli. Figure 8 shows the trial structure and stimuli used in Experiment 4.

\section{Experimental design}

We grouped the stimuli such that " $H$ " flankers were paired with "S" flankers (HHHHH, HHCHH, SSSSS, SSKSS) and "C" flankers were paired with " $\mathrm{K}$ " flankers (CCCCC, $\mathrm{CCHCC}, \mathrm{KKKKK}, \mathrm{KKSKK})$. One group was randomly selected as the standard flankers for each subject and the other was the oddball (counterbalanced across subjects). Within each group, its four stimuli occurred equiprobably. Note that within each group, there was one congruent stimulus for which the correct response was a left key press and one for which the correct response was a right key press. Thus, not only were left and right responses equiprobable, but they were equiprobable within a congruency context.

We tested two levels of probability of standard and oddball distractors: $0.50 / 0.50$ and $0.90 / 0.10$. In the $0.50 / 0.50$ condition, each flanker configuration occurred with probability 0.25 . In the $0.90 / 0.10$ condition, standard flankers occurred with probability 0.45 and oddball flankers occurred with probability 0.05 . By controlling the relationship between target identity and congruency, we halved the frequency with which any flanker configuration occurred.

Instructions and practice trials were identical to those in Experiments 2 and 3. Following this, subjects completed 140 trials in the $0.50 / 0.50$ probability condition (of which we analyzed the final 80 trials, to give subjects ample opportunity to acclimate to the task), followed by four experimental blocks of $0.90 / 0.10$ probability each with 80 trials. The standard and oddball stimuli groups were held constant across the four blocks.

\section{Analyses}

Data processing and analyses were generally identical to those on Experiments 2 and 3, with identical trial exclusion criteria. Our final data set comprised 48 subjects who gave valid responses on more than 340 of 400 trials (mean $=392$ trials, range: $346-400$ ). The percentage of excluded trials based on trial conditions was standard congruent, $2.20 \%$; oddball-congruent, $1.85 \%$; standardincongruent, 1.91\%; oddball-incongruent, $2.20 \%$.

\section{Results}

We modified Experiment 1 by using two sets of letter stimuli and two standard to oddball flanker probability ratios: $0.50 /$ 0.50 and $0.90 / 0.10$. Figure 9 summarizes response accuracy and median response times on correct trials.

Overall, accuracy was highest on standard-congruent trials $(0.936, S D=0.045)$, next highest on oddball-congruent trials $(0.926, S D=0.064 \mathrm{~ms})$, third highest on standard-incongruent trials $(0.909, S D=0.067)$, and lowest on oddball-incongruent trials $(0.898, S D=0.066)$. Subjects also responded more quickly on congruent than on incongruent trials. Overall, responses were fastest on standard-congruent trials (472 $\mathrm{ms}, S D=114 \mathrm{~ms}$ ), next fastest on oddball-congruent trials (478 ms, $S D=106 \mathrm{~ms})$, followed by standard-incongruent 
a

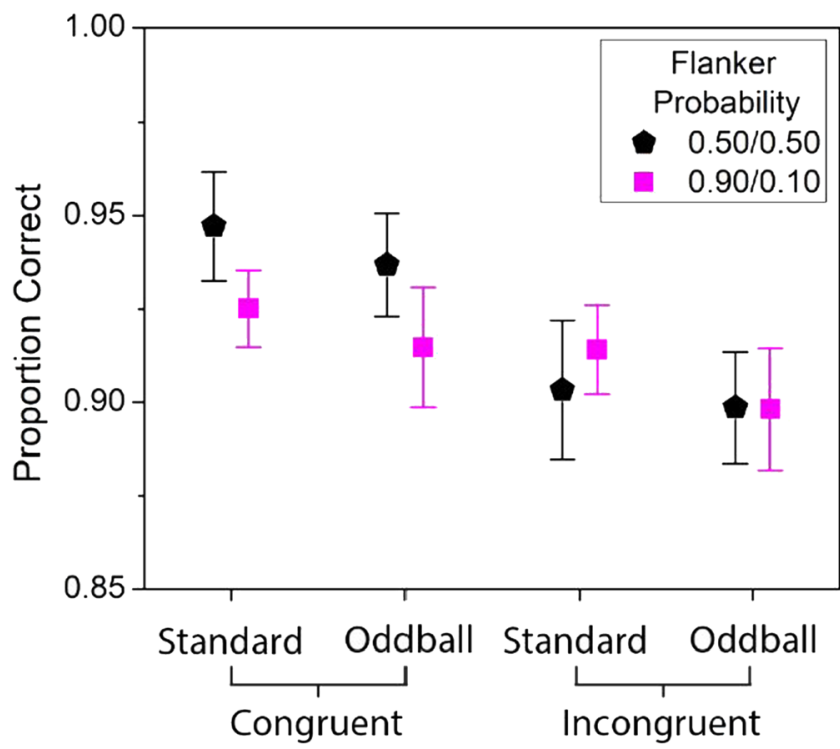

Fig. 9 Accuracy and response time for all conditions. a Proportion correct for each trial type by flanker probability level. b Median response time for correct responses. Each condition includes data from

trials $(484 \mathrm{~ms}, S D=96 \mathrm{~ms})$, and oddball-incongruent trials ( $503 \mathrm{~ms}, S D=117 \mathrm{~ms}$ ). Across the board, response times for $0.90 / 0.10$ blocks were approximately $25-35 \mathrm{~ms}$ faster than response times for the $0.50 / 0.50$ block, again likely due to block order effects.

Oddball-incongruent response times were significantly faster on $0.90 / 0.10$ blocks than on $0.50 / 0.50$ blocks, paired $t(47)=2.361, p=.0224$, but there was no difference in proportion correct, paired $t(47)=.032, p=.9749$. Similarly, standard incongruent response times were significantly faster on $0.90 / 0.10$ blocks than on $0.50 / 0.50$ blocks, paired $t(47)=$ $3.283, p=.0019$, but there was no significant difference in proportion correct, paired $t(47)=1.078, p=0.2867$. Further, there was no significant difference between response times of standard incongruent and oddball-incongruent trials in 0.50 / 0.50 blocks, paired $t(47)=1.778, \mathrm{p}=.0819$. Finally, we confirmed that there were no consistent accuracy or response time differences between configurations within the same condition (e.g., CCHCC and KKSKK, or HHHHH and SSSSS), and thus do not report them here.

\section{Discussion}

In Experiment 4, we used two sets of letter stimuli to avoid a confound in our previous studies, for which stimulus congruency was predictive of target response. Indeed, the striking difference in performance between $0.50 / 0.50$ and $0.90 / 0.10$ oddball-incongruent trials was no longer observed. As in our earlier experiments, responses to congruent trials were faster and slightly more accurate than those to incongruent trials (see Fig. 9a). Response times were markedly longer on $0.50 / 0.50$ b

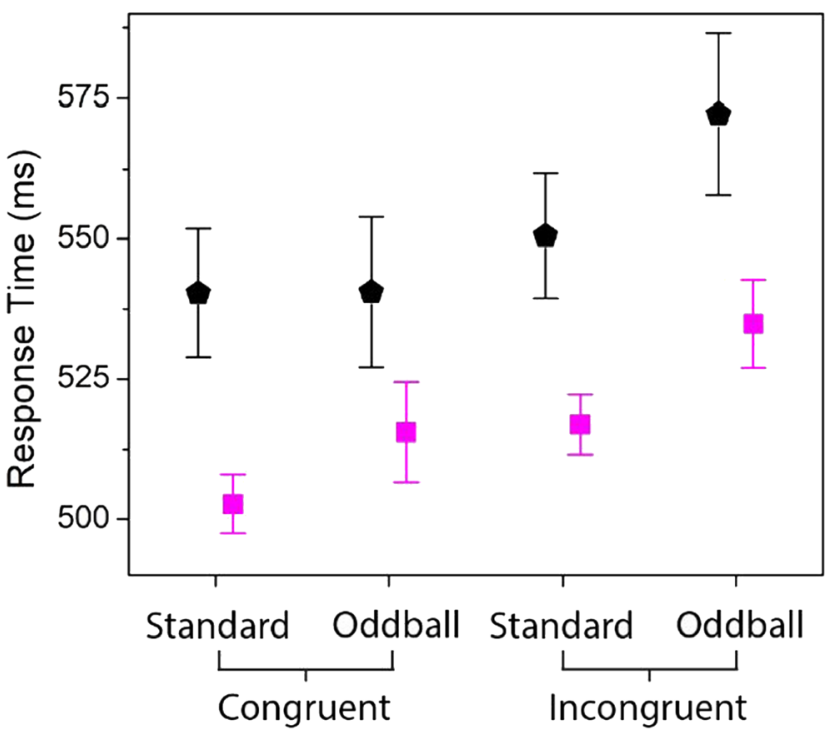

two different configurations for each flanker probability level. Error bars are twice the repeated-measures standard error of the mean

trials than $0.90 / 0.10$ trials (see Fig. $9 b$ ), and noticeably longer than in the previous experiments (see Figs. 2b-c, and 6d). There was no evidence of the oddball-incongruency effect that we observed in other studies.

We think at least three factors contributed to the pattern of results in this experiment. First, doubling the stimuli set size required subjects to learn a more complex target-response mapping, making the task more challenging and possibly requiring subjects to use a thoughtful, deliberate mode of response rather than a more automatic one. The slower response times are consistent with this account, as are the differences in response time between $0.50 / 0.50$ and $0.90 / 0.10$ blocks. As subjects slow down to cope with the complexity of the target-to-key-press mapping, attentional selection processes have more time to correctly segregate the target stimulus from the surrounding clutter. The quite-small congruency effects observed in this experiment are consistent with this account.

Second, doubling the stimuli set halved the frequency with which each configuration occurred. standard trials on $0.90 / 0.10$ blocks are now approximately as probable as on $0.50 / 0.50$ blocks in the earlier studies. This increase in stimulus exemplars and decrease in stimulus frequency may have prevented the formation of expectations powerful enough to significantly affect responses (as in a resource model; Bays \& Husain, 2008; Joseph, Teki, Kumar, Husain, \& Griffiths, 2016; Luck \& Vogel, 2013). Consistent with our findings, past work has also found that larger sets of stimuli lead to reduced congruency effects in the flanker task (Blais \& Verguts, 2012).

Finally, it is possible that the effects seen in the first three studies are entirely due to the relationship between congruency and target identity. It may be that rather than attentionally 
selecting the target, subjects are learning a response contingent to the entire configuration. Here, we tested one experimental design that found no oddball-incongruent effect when congruency and correct response were balanced; however, we suspect that the increased difficulty of this task variant makes it qualitatively different from the classic chevrons task. Next steps toward disentangling these aspects might include a design with two sets of intrinsically directional stimuli, or with targets drawn from a wider set of stimuli than flankers.

\section{General Discussion}

The aim of these experiments was to understand how people form expectations according to the probability of specific visual distractors. We manipulated the relative probabilities of distractor elements within the Eriksen flanker task and measured the effects of both global (block-level) statistical structure and local (trial-by-trial) patterns. We observed a logarithmic-scale relationship between flanker probability and accuracy (see Fig. 3). Local trial-by-trial structure and block-level statistics both affected performance (see Figs. 2, 4, 6, and 7). Both chevron and letter stimuli elicited similar patterns of results, with the effects of both globally derived and locally derived expectations decreasing in the latter case (see Fig. 6), Finally, we found that when we doubled the number of stimuli, which both increased task difficulty and decreased the strength of expectations, these effects vanished (see Fig. 9). Taken as a whole, our results provide evidence that expectations, formed via statistical patterns among to-be-ignored aspects of the stimuli, facilitate performance in the flanker task.

Expectations, even implicit ones, about forthcoming events or sensory inputs strongly shape behavior (Schultz, Dayan, \& Montague, 1997; Summerfield \& Egner, 2009), and can develop, persist, and transform over multiple time scales (Meyer \& Bucci, 2016; Noonan et al., 2018). People are biased to perceive stimuli that are more likely in a given setting, at levels from simple features to complex interrelationships (Bar, 2009; Biederman, 1981; Chalk, Seitz, \& Seriès, 2010; Greene, 2013; Slevc \& Okada, 2015). Expectation also facilitates attention and reduces the effects of conflict (Bugg \& Smallwood, 2016; Nöstl, Marsh, \& Sörqvist, 2012). Expectation-mediated distractor inhibition, one mechanism that may underly our results, facilitates target selection and appropriate responses (Awh et al., 2003; Chao, 2011; Noonan et al., 2018; Ruff \& Driver, 2006; van Moorselaar \& Slagter, 2019).

There are several processes by which oddball-incongruent flankers could disrupt performance on this task. Unexpected or poorly predicted events reliably capture attention (Wills, Lavric, Croft, \& Hodgson, 2007) even in a to-be-ignored location or sensory modality (Nöstl et al., 2012; Parmentier, Elford, Escera, \& Andre, 2008). An unexpected flanker configuration may "reset" an attentional spotlight to encompass the entire display, leading to response errors (White, Ratcliff, \& Starns, 2011). Congruency effects may also be explained by the activation of conflicting response schemas, which requires that the subject must resolve the conflict to generate an appropriate response (Botvinick et al., 2001; Gratton, Coles, \& Donchin, 1992; Sanders \& Lamers, 2002). Expectationmediated inhibition of probable flankers would facilitate performance under either of these accounts.

Several possible alternative explanations for our results can be ruled out. The performance deficit in the presence of lowprobability flankers cannot be explained by subjects' potential anticipation of motor responses or target orientations (as in Mayr et al., 2003; Wendt \& Luna-Rodriguez, 2009). Within each block, regardless of flanker probability structure, the target orientations were left-pointing and right-pointing on exactly half of trials. Similarly, the observed effects cannot be attributed to increased cognitive control following incongruent trials (Bugg, Diede, Cohen-Shikora, \& Selmeczy, 2015; Gratton et al., 1992), because congruent and incongruent configurations also each occurred on exactly half of trials. However, repeated distractors can modulate flanker congruency effects (Davelaar, 2013), and the likelihood of repeated distractors varies with flanker probability. Subjects' expectations about distractors may thus manifest in part as general adjustments in cognitive control.

We have described conceptually this task as one in which subjects must discriminate targets from flankers within a cluttered display. It is possible that subjects are, instead, learning to represent the stimulus displays as gestalt configurations and responding by identifying each possible configuration. That is, subjects may have learned than congruent patterns are usually associated with a left (or right) key press, and incongruent patterns with the other. To balance flanker identity and target identity in Experiments 1, 2, and 3, our design confounded congruency and correct response; in Experiment 4 , when congruency and response were balanced, we did not see an oddball-incongruent effect. However, we believe the gestalt configuration account is inadequate to explain several aspects of our findings. First, if congruency-response associations formed as a result of learning configuration-target patterns, we would expect to see at least some decrease in accuracy on oddball-congruent trials as they become rarer. However, in Experiments 1-3, we only see a decrease on oddball-incongruent trials (Figs. 2, and 6), suggesting that this specific configuration, and not the oddball-congruent configuration, is difficult. Further, in Experiments 2 and 3, we observed that the oddball-incongruent effect was smaller for letter stimuli than for chevron stimuli. Again, this suggests that specific stimulus configurations are challenging, and that the difficulty of a specific configuration depends on the geometric dissimilarity between targets and flankers - which is compatible with the target selection account. Finally, the results of Experiment 4 alone are not sufficient to firmly conclude a role 
for congruency-response associations; there are several alternatives that we cannot yet rule out. It is possible that multiple cognitive mechanisms contributed to our results, such that there would be a benefit from further investigations focused on definitively showing the underlying mechanism(s).

Expectation-mediated distractor inhibition depends, of course, on a form of distractor learning. A primary aim of our study was to shed light on the timescales over which expectations develop. We found that expectations are shaped by local sequences of trials and block-level statistical structure. In Experiment 2, the length of a preceding run of standard flankers was a significant predictor of performance on a subsequence oddball-incongruent trial. Similarly, in a visual search task, expectations change rapidly in response to an unexpected distractor; when a distractor repeats, the rate of learning slows (van Moorselaar \& Slagter, 2019). This suggests that potential expectations about distractors can be continuously adjusted in response to recent events. The fluidity of expectations is conceptually compatible with numerous proposed mechanisms of stimuli representation, such as multiscale associative learning (Blais \& Verguts, 2012; Colzato, Raffone, \& Hommel, 2006; Davelaar, 2013) or biases in perception (Noonan et al., 2018).

Our results extend these findings by examining the effects of statistical regularities at different scales, both trial-by-trial structure and block-level probabilities. On Experiments 1 and 2 , which used chevron stimuli, we found an interaction between block-level statistical structure and local runs of repeated flankers. We did not find a corresponding interaction on Experiment 3, which used letter stimuli. The increased response times in Experiment 3 likely reflect more demanding mapping from stimulus to response, which in turn may hinder the formation or reduce the effects of distractor expectations. We suspect that the automaticity of responses to chevron stimuli facilitates both the development of these expectations and subjects' reliance on them to perform the task quickly and accurately. Informal debriefing of subjects led to many reports of response errors even as they recognized the correct response. The increased response times in Experiment 3 suggest that automaticity may play a smaller role than in Experiment 2, and could explain the smaller oddball-incongruency effect. In Experiment 4, the lack of an oddball-incongruency effect and the increase in response times also supports the notion that automaticity plays a role in expectation-mediated flanker task performance. In Experiment 1, we also found that runs of repeated targets decreased accuracy more than did runs of repeated flankers, consistent with an established result that sequence effects are strongest for attended features (Blais \& Verguts, 2012; Gratton et al., 1992).

Some of the most interesting prior work on distractor learning has looked at time courses much longer than those in our experiment. Distractor learning in a texture discrimination task developed over the course of days (Karni \& Sagi,
1991); another study observed dissociations between learning on different time scales (i.e., days vs. sessions; see Dantec, Melton, \& Seitz, 2012; see Fine \& Jacobs, 2002, for a thorough review of perceptual learning). These results suggest that different aspects of learning and expectation forming may occur at different timescales. In the current work, subjects were able to develop expectations quickly and implicitly (when asked an open-ended question about trends in trial structure, some subjects recognized repetitions of trials, though none could identify specific trends in distractor probability), suggesting that they developed implicit expectations, similar to what occurs in other forms of contextual learning. Relevant recent work studying attention and inhibition in the flanker task trained subjects to inhibit distractors or better discriminate targets, finding that inhibition training resulted in greater improvements in performance (Melara, Singh, Hien, Russo, \& Maier, 2018). While our results were obtained over timescales too small to be comparable, an intriguing avenue of research could build on our findings to elucidate the relationship between probabilistically driven distractor learning across a wide range of timescales.

Our experiments and results emphasize how expectations influence attentional selection in the presence of stimulus and response conflict. We found that flanker expectations form in response to statistical regularities across multiple timescales, and that their effects are strongest when stimuli are geometrically similar and subjects are able to respond to trials quickly. Improbable distractors disrupt performance, most likely via a form of attentional capture. This work expands our understanding of the role that expectations can play in complex cognitive settings, and illuminates the multiple, nested factors that can shape such expectations.

Acknowledgements This study was supported by a National Institutes of Deafness and Communication Disorders grant (R01 DC013825). The authors would like to thank Audra Irvine for assistance with data collection and demographics analysis.

Author contributions Original idea: A.N.; Ran Experiments \& Data Processing: E.B.; Data Interpretation: A.N., B.S.C., E.B.; Wrote manuscript: A.N., B.S.C., E.B.

\section{Compliance with ethical standards}

Open practices statement The data reported here are available for download at https://osf.io/akcgd/. Experiment code is available upon request to the authors. This study was not preregistered.

\section{References}

Anwyl-irvine, A. L. (2020). Gorilla in our midst: An online behavioral experiment builder. Behavior Research Methods, 52, 388-407.

Arman, A. C., Ciaramitaro, V. M., \& Boynton, G. M. (2006). Effects of feature-based attention on the motion aftereffect at remote locations. 
Vision Research, 46(18), 2968-2976. doi:https://doi.org/10.1016/j. visres.2006.03.003

Aron, A. R. (2011). From reactive to proactive and selective control: Inappropriate responses. Biological Psychiatry, 69(12), e55-e68. doi:https://doi.org/10.1016/j.biopsych.2010.07.024

Awh, E., Matsukura, M., \& Serences, J. T. (2003). Top-down control over biased competition during covert spatial orienting. Journal of Experimental Psychology: Human Perception and Performance, 29(1), 52-63. doi:https://doi.org/10.1037/0096-1523.29.1.52

Bar, M. (2009). The proactive brain: Memory for predictions. Philosophical Transactions of the Royal Society B, 1235-1243. doi:https://doi.org/10.1098/rstb.2008.0310

Bays, P. M., \& Husain, M. (2008). Dynamic shifts of limited working memory resources in human vision. Science, 321(5890), 851-854.

Biederman, I. (1981). On the semantics of a glance at a scene. In M. Kubovy \& J. R. Pomerantz (Eds.), Perceptual organization (pp. 213-253). Hillsdale, NJ: Erlbaum.

Blais, C., \& Verguts, T. (2012). Increasing set size breaks down sequential congruency: Evidence for an associative locus of cognitive control. Acta Psychologica, 141(2), 133-139. doi:https://doi.org/10. 1016/j.actpsy.2012.07.009

Botvinick, M. M., Braver, T. S., Barch, D. M., Carter, C. S., \& Cohen, J. D. (2001). Conflict monitoring and cognitive control. Psychological Review, 108(3), 624-652. doi:https://doi.org/10.1037//0033-295X. I08.3.624

Braver, T. S. (2012). The variable nature of cognitive control: A dual mechanisms framework. Trends in Cognitive Sciences, 16(2), 105-112. doi:https://doi.org/10.1016/j.tics.2011.12.010

Bugg, J. M., Diede, N. T., Cohen-Shikora, E. R., \& Selmeczy, D. (2015). Expectations and experience: Dissociable bases for cognitive control? Journal of Experimental Psychology: Learning, Memory, and Cognition, 41(5), 1349-1373.

Bugg, J. M., \& Smallwood, A. (2016). The next trial will be conflicting! Effects of explicit congruency pre-cues on cognitive control. Psychological Research, 80(1), 16-33. doi:https://doi.org/10.1007/ s00426-014-0638-5

Carrasco, M. (2011). Visual attention: The past 25 years. Vision Research, 51(13), 1484-1525. doi:https://doi.org/10.1016/j.visres. 2011.04.012

Chalk, M., Seitz, A. R., \& Seriès, P. (2010). Rapidly learned stimulus expectations alter perception of motion. Journal of Vision, 10(8), 118. doi:https://doi.org/10.1167/10.8.2.Introduction

Chao, H. (2011). Active inhibition of a distractor word: The distractor precue benefit in the Stroop color-naming task. Journal of Experimental Psychology: Human Perception and Performance, 37(3), 799-812. doi:https://doi.org/10.1037/a0022191

Cohen, J. D., Servan-Schreiber, D., \& Mcclelland, J. L. (1992). A parallel distributed processing approach to automaticity. The American Journal of Psychology, 105(2), 239-269.

Colzato, L. S., Raffone, A., \& Hommel, B. (2006). What do we learn from binding features? Evidence for multilevel feature integration. Journal of Experimental Psychology: Human Perception and Performance, 32(3), 705-716. doi:https://doi.org/10.1037/00961523.32.3.705

Cousineau, D. (2005). Confidence intervals in within-subject designs: A simpler solution to Loftus and Masson's method. Tutorials in Quantitative Methods for Psychology, 1(1), 42-45. doi:https://doi. org/10.20982/tqmp.01.1.p042

Davelaar, E. J. (2013). When the ignored gets bound: Sequential effects in the flanker task. Frontiers in Psychology, 3(January), 1-13. doi: https://doi.org/10.3389/fpsyg.2012.00552

Eriksen, B., \& Eriksen, C. W. (1974). Effects of noise letters upon the identification of a target letter in a nonsearch task. Perception \& Psychophysics, 16(1), 143-149.

Eriksen, C. W., \& Eriksen, C. W. (1995). The flankers task and response competition: A useful tool for investigating a variety of cognitive problems. Visual Cognition, 2(2/3), 101-118. doi:https://doi.org/10. 1080/13506289508401726

Ferdinand, N. K., Mecklinger, A., \& Kray, J. (2008). Error and deviance processing in implicit and explicit sequence learning. Journal of Cognitive Neuroscience, 20(4), 629-642. doi:https://doi.org/10. 1162/jocn.2008.20046

Fine, I., \& Jacobs, R. A. (2002). Comparing perceptual learning across tasks: A review. Journal of Vision, 2(5), 190-203.

Foster, J. J., Bsales, E. M., \& Awh, E. (2020). Covert spatial attention speeds target individuation. Journal of Neuroscience, 40(13). doi: https://doi.org/10.1101/838557

Ghinescu, R., Schachtman, T. R., Ramsey, A. K., Gratton, G., \& Fabiani, M. (2016). Conflict adaptation and cue competition during learning in an Eriksen flanker task. PLOS ONE, 1-19. doi:https://doi.org/10. 1371/journal.pone.0167119

Goschy, H., Bakos, S., Müller, H. J., \& Zehetleitner, M. (2014). Probability cueing of distractor locations: both intertrial facilitation and statistical learning mediate interference reduction. Frontiers in Psychology, 5(1195), 1-11. doi:https://doi.org/10.3389/fpsyg.2014. 01195

Gratton, G., Coles, M. G. H., \& Donchin, E. (1992). Optimizing the use of information: Strategic control of activation of responses. Journal of Experimental Psychology: General, 121(4), 480-506.

Greene, M. R. (2013). Statistics of high-level scene context. Frontiers in Psychology, 4(October), 1-31. doi:https://doi.org/10.3389/fpsyg. 2013.00777

Hajcak, G., \& Foti, D. (2008). Errors are aversive defensive motivation and the error-related negativity. Psychological Science, 19(2), 103108.

James, W. (1890). The principles of psychology. New York, NY: Dover.

Johnston, J. C., \& Pashler, H. (1990). Close binding of identity and location in visual feature perception. Journal of Experimental Psychology: Human Perception and Performance, 16(4), 843-856.

Joseph, S., Teki, S., Kumar, S., Husain, M., \& Griffiths, T. D. (2016). Resource allocation models of auditory working memory. Brain Research, 1640(Pt. B), 183-192. doi:https://doi.org/10.1016/j. brainres.2016.01.044

Karni, A., \& Sagi, D. (1991). Where practice makes perfect in texture discrimination: Evidence for primary visual cortex plasticity. Proceedings of the National Academy of Sciences of the United States of America, 88(June), 4966-4970.

Keller, A. S., Payne, L., \& Sekuler, R. (2017). Characterizing the roles of alpha and theta oscillations in multisensory attention. Neuropsychologia, 99, 48-63. doi:https://doi.org/10.1016/j. neuropsychologia.2017.02.021

Keller, A. S., \& Sekuler, R. (2015). Memory and learning with rapid audiovisual sequences. Journal of Vision, 15, 1-18. doi:https://doi. org/10.1167/15.15.7.doi

Dantec, C. C. Le, Melton, E. E., \& Seitz, A. R. (2012). A triple dissociation between learning of target, distractors, and spatial contexts. Journal of Vision, 12, 1-12. doi:https://doi.org/10.1167/12.2.5. Introduction

Leber, A. B., Gwinn, R. E., Hong, Y., \& O’Toole, R. J. (2016). Implicitly learned suppression of irrelevant spatial locations. Psychonomic Bulletin \& Review, 1873-1881. doi:https://doi.org/10.3758/ s13423-016-1065-y

Luck, S. J., \& Vogel, E. K. (2013). Visual working memory capacity: From psychophysics and neurobiology to individual differences. Trends in Cognitive Sciences, 17(8), 391-400. doi:https://doi.org/ 10.1016/j.tics.2013.06.006

Mayr, U., Awh, E., \& Laurey, P. (2003). Conflict adaptation effects in the absence of executive control. Nature Neuroscience, 6(5), 6-8. doi: https://doi.org/10.1038/nn1051

Melara, R. D., Singh, S., Hien, D. A., Russo, F., Di, \& Maier, M. E. (2018). Neural and behavioral correlates of attentional inhibition training and perceptual discrimination training in a visual flanker 
task. Frontiers in Human Neuroscience, 12(May), 1-15. doi:https:// doi.org/10.3389/fnhum.2018.00191

Meyer, H. C., \& Bucci, D. J. (2016). Neural and behavioral mechanisms of proactive and reactive inhibition. Learning \& Memory, 23, 504 514.

Michalka, S. W., Kong, L., Rosen, M. L., Shinn-Cunningham, B. G., \& Somers, D. C. (2015). Short-term memory for space and time flexibly recruit complementary sensory-biased frontal lobe attention networks. Neuron, 87(4), 882-892. doi:https://doi.org/10.1016/j. neuron.2015.07.028

Neumann, E., \& Deschepper, B. G. (1991). Costs and benefits of target activation and distractor inhibition in selective attention. Journal of Experimental Psychology: Learning, Memory, and Cognition, 17(6), 1136-1145.

Noonan, M. P., Crittenden, B. M., Jensen, O., \& Stokes, M. G. (2018). Selective inhibition of distracting input. Behavioural Brain Research, 355(April 2017), 36-47. doi:https://doi.org/10.1016/j. bbr.2017.10.010

Nöstl, A., Marsh, J. E., \& Sörqvist, P. (2012). Expectations modulate the magnitude of attentional capture by auditory events. PLOS ONE, 7(11). doi:https://doi.org/10.1371/journal.pone.0048569

Noyce, A., \& Sekuler, R. (2014). Oddball distractors demand attention: Neural and behavioral responses to predictability in the flanker task. Neuropsychologia , 65, 18-24. doi:https://doi.org/10.1016/j. neuropsychologia.2014.10.002

Parmentier, F. B. R., Elford, G., Escera, C., \& Andre, P. (2008). The cognitive locus of distraction by acoustic novelty in the crossmodal oddball task. Cognition, 106(1), 408-432. doi:https://doi. org/10.1016/j.cognition.2007.03.008

Payne, L., \& Sekuler, R. (2014). The importance of ignoring: Alpha oscillations protect selectivity. Current Directions in Psychological Science, 23(3), 171-177. doi:https://doi.org/10. $1177 / 0963721414529145$

Ruff, C. C., \& Driver, J. (2006). Attentional preparation for a lateralized visual distractor: Behavioral and fMRI evidence. Journal of Cognitive Neuroscience, 18(4), 522-538. doi:https://doi.org/10. 1162/jocn.2006.18.4.522

Russeler, J., Kuhlicke, D., \& Munte, F. T. (2003). Human error monitoring during implicit and explicit learning of a sensorimotor sequence. Neuroscience Research, 47, 233-240. doi:https://doi.org/10.1016/ S0168-0102(03)00212-8

Sanders, A. F., \& Lamers, J. M. (2002). The Eriksen flanker effect revisited. Acta Psychologica, 109(1), 41-56. doi:https://doi.org/10. 1016/s0001-6918(01)00048-8

Schmidt, P. A., \& Dark, V. J. (1998). Attentional processing of "unattended" flankers: Evidence for a failure of selective attention. Perception \& Psychophysics, 60(April 1993), 227-238.

Schultz, W., Dayan, P., \& Montague, P. R. (1997). A neural substrate of prediction and reward. Science, 275(5306), 1593-1599.

Servant, M., \& Logan, G. D. (2019). Dynamics of attentional focusing in the Eriksen flanker task. Attention, Perception, \& Psychophysics, $81,2710-2721$.
Slevc, L. R., \& Okada, B. M. (2015). Processing structure in language and music: A case for shared reliance on cognitive control. Psychonomic Bulletin \& Review, 22(3), 637-652. doi:https://doi. org/10.3758/s13423-014-0712-4

Summerfield, C., \& Egner, T. (2009). Expectation (and attention) in visual cognition. Trends in Cognitive Sciences, 13(9), 403-409. doi:https://doi.org/10.1016/j.tics.2009.06.003

Tipper, S. P. (2001). Does negative priming reflect inhibitory mechanisms? A review and integration of conflicting views. The Quarterly Journal of Experimental Psychology, 2, 321-343. doi: https://doi.org/10.1080/0272498004200018

Tomat, M., Wendt, M., Luna, A., Michael, R., \& Thomas, S. (2020). Target - distractor congruency: sequential effects in a temporal flanker task. Psychological Research, 84(2), 292-301. doi:https:// doi.org/10.1007/s00426-018-1061-0

Treue, S., \& Martínez Trujillo, J. C. (1999). Feature-based attention influences motion processing gain in macaque visual cortex. Nature, 399(6736), 575-579.

Tseng, P., Tzengô, O. J. L., \& Hungô, D. L. (2011). Probabilities in implicit learning. Perception, 40, 822-829. doi:https://doi.org/10. 1068/p6833

van Moorselaar, D. (2020). Inhibition in selective attention. Annals of the New York Academy of Sciences, 1464(1), 204-221. doi:https://doi. org/10.1111/nyas.14304

van Moorselaar, D., \& Slagter, H. A. (2019). Learning what is irrelevant or relevant: Expectations facilitate distractor inhibition and target facilitation through distinct neural mechanisms. The Journal of Neuroscience, 39(35), 6953-6967. doi:https://doi.org/10.1523/ JNEUROSCI.0593-19.2019

Wang, B., \& Theeuwes, J. (2018). How to inhibit a distractor location? Statistical learning versus active, top-down suppression. Attention, Perception, \& Psychophysics, 860-870.

Wendt, M., \& Luna-Rodriguez, A. (2009). Conflict-frequency affects flanker interference: Role of stimulus-ensemble-specific practice and flanker-response contingencies. Experimental Psychology, 56(3), 206-217. doi:https://doi.org/10.1027/1618-3169.56.3.206

White, C. N., Ratcliff, R., \& Starns, J. J. (2011). Diffusion models of the flanker task: Discrete versus gradual attentional selection. Cognitive Psychology, 63(4), 210-238. doi:https://doi.org/10.1016/j. cogpsych.2011.08.001

Wills, A. J., Lavric, A., Croft, G. S., \& Hodgson, T. L. (2007). Predictive learning, prediction errors, and attention: Evidence from eventrelated potentials and eye tracking. Journal of Cognitive Neuroscience, 19(5), 843-854.

Wühr, P., Frings, C., \& Heuer, H. (2018). response preparation with reliable cues decreases response competition in the flanker task. Experimental Psychology, 65(5), 286-296.

Publisher's note Springer Nature remains neutral with regard to jurisdictional claims in published maps and institutional affiliations. 